\title{
3D Interventional Imaging with 2D X-Ray Detectors
}

\author{
L. Desbat ${ }^{1}$, G. Champleboux ${ }^{1}$, M. Fleute ${ }^{1}$, P. Komarek ${ }^{1}$, C. Mennessier ${ }^{1}$, \\ B. Monteil ${ }^{2}$, T. Rodet ${ }^{1}$, P. Bessou ${ }^{3}$, M. Coulomb ${ }^{4}$, and G. Ferretti ${ }^{4}$ \\ 1 TIMC-IMAG, IAB, Faculté de Médecine, UJF, 38706 La Tronche France \\ Laurent.Desbat@imag.fr \\ http://www-timc.imag.fr/cami/ \\ 2 TRIXELL, Parc d'Activités Centr'Alp, 38430 Moirans France \\ 3 Neuroradiology department of the Grenoble University Hospital \\ 38000 Grenoble France \\ 4 Radiology and Medical Imaging department of the Grenoble University Hospital \\ 38000 Grenoble France
}

\begin{abstract}
Pre-operative images, such as CT or MRI, are often necessary for CAMI. However, they could be replaced by interventional 3D reconstruction from $2 \mathrm{D}$ x-ray sensors. $3 \mathrm{D}$ reconstruction from classical image amplifiers needs the correction of geometric distortions due to the magnetic fields. We investigate new calibration marker schemes exploiting spectral properties of the x-ray transform. According to Shannon theory, no information is lost with these new schemes, even if the markers can be seen in each image. Numerical experiments from both phantom and real data are provided.
\end{abstract}

\section{Introduction}

Computer Assisted Medical Intervention needs numerical information on the patient. This information is treated by computers in order to propose some optimal strategy for the intervention. Then, the surgeon can be assisted during the operation. Medical imaging is an essential source of information available on the patient. Nowadays MRI and CT lead to sufficiently precise information for planning a wide range of interventions. MRI has the essential advantage of being non-invasive. Nevertheless, for complex bone surgery (traumatology, orthopedy), $\mathrm{CT}$ and radiologic images remain necessary because of their incomparable quality. However, MRI and CT systems are rather slow and not ergonomic. They are mainly used as pre-operative imaging devices. Moreover, pre-operative images need to be registered with the patient during the intervention. This implies to acquire information on the patient during the intervention for this registration. Intra-operative imaging systems just need to be calibrated. They can be used for the planning but also to guide the surgeon. They offer the possibility to control and optimize dynamically the intervention. They are more flexible. Their use can be optimized for each intervention protocol. Thus, interventional imaging is a natural trend. 
Ultrasound and x-ray systems coupled to a localizer are already used during interventions in order to acquire 3D information [3,12]. For some applications, a limited number of acquisitions is sufficient. Moreover, a priori information can be used to improve the identification of the unknown parameters [8]. In this work we consider $3 \mathrm{D}$ reconstruction of attenuation coefficients from image intensifiers. This can produce pre-operative 3D images for planning but also interventionnal 3D images for controling. Since the beginning of 1980s, 3D reconstruction techniques from conical x-ray projections have been greatly improved $[7,11,5,14]$. Reconstructions of high contrast structures from few projections acquired on image intensifiers have been proposed [15,4]. Image intensifiers are widespread, relative low cost imaging system already available in most surgical rooms. However, image intensifiers suffer from geometric distortions due to magnetic fields. These distortions must be corrected before the reconstruction. Because the image intensifier distortion depends on the surrounding magnetic field, a dynamic distortion correction needs to be implemented. Indeed, the correction depends on the position and orientation of the image intensifier. Because the modeling of these distortions is very difficult, opaque markers are usually used to estimate the distortion. For a static acquisition, a first image with markers is generally acquired. Then the distortion is estimated. The distortion correction is finally applied to a second image without markers (see [1] for details). This method can hardly be implemented for dynamic acquisition: rapidly removable marker grids are not available and seem difficult to be designed for a rate of more than 10 images per seconds. Moreover, this approach implies a significant increase of $\mathrm{x}$-ray dose on the patient.

In section 2, we first propose a calibration marker geometry that can stay on the image amplifier: it enables a dynamic distorsion correction of 3D tomographic acquisitions with almost no loss of information according to Shannon theory. Spectral properties of the 3D projection data are exploited.

\section{3D Reconstruction from Image Intensifiers}

For interventional 3D reconstructions from image intensifiers, we need to collect a set of x-ray projections, regularly spaced around the patient. In this paper, we only consider the simplest trajectory of the source and detector around the patient, i.e., one circle. In this work we show that spectral properties of the data can be exploited to design a calibration marker geometry, which is "invisible" in the reconstruction. We first present the idea in 2D tomography. Spectral properties of the 2D Radon transform are shown in section 2.1. A generalization to the $3 \mathrm{D}$ x-ray transform is then presented. The main result is that standard sampling schemes (equally spaced projection angles, equally distant sampling of the detector) are redundant. Thus, we can recover the data covered by opaque markers for particular (interlaced) marker distribution schemes. In section 2.2, we present a fast Fourier interpolation formula in 2D. In section 2.3, we present $3 \mathrm{D}$ reconstructions from data containing interlaced markers. They are as good 
as $3 \mathrm{D}$ reconstructions from data without marker. This approach is then tested on real data.

\subsection{Data Spectum and Efficient Sampling}

Let $g(y)$ be an $n$-dimensional function to be sampled (for simplicity let $g \in$ $\mathcal{S}\left(\mathbb{R}^{n}\right)$, the Schwartz space of $\mathbb{R}^{n}$ ), let $\mathbf{K}$ be the essential support of its Fourier transform $\hat{g}(\xi)=(2 \pi)^{-1 / 2} \int_{\mathbb{R}^{n}} g(x) e^{-i x \cdot \xi} d x$, more precisely $\int_{\xi \notin \mathbf{K}}|\hat{g}(\xi)| d \xi$ is supposed to be almost zero. The non overlapping Shannon condition associated to a sampling scheme generated by the non singular matrix $W$, i.e., the sampling set $\left\{W l, l \in \mathbb{Z}^{n}\right\}$, is that the sets $\mathbf{K}$ translated on the reciprocal scheme, i.e., $\mathbf{K}+2 \pi W^{-1^{t}} l, l \in \mathbb{Z}^{2}$, do not overlap. If this condition is satisfied, then $g(y), y \in \mathbb{R}^{n}$ can be estimated from the Fourier interpolation formula:

$$
\left(S_{W} g\right)(y)=(2 \pi)^{-n / 2}|\operatorname{det} W| \sum_{k \in \mathbb{Z}^{n}} g(W k) \hat{\chi}_{\mathbf{K}}(W k-y)
$$

with the error estimate

$$
\left\|S_{W} g-g\right\|_{\infty} \leq 2(2 \pi)^{-n / 2} \int_{\xi \notin \mathbf{K}}|\hat{g}(\xi)| d \xi .
$$

Thus, geometrical properties of the set $\mathbf{K}$ can be exploited for the choice of $W$ satisfying the non-overlapping Shannon conditions. Efficient sampling is based on the choice of $W$ yielding the most compact non-overlapping conditions. In this case $\operatorname{det}\left(W^{-1^{t}}\right)$ is minimal, equivalently $\operatorname{det}(W)$ is maximal, hence the sampling scheme is the sparsest among those satisfying the Shannon conditions. Nonefficient sampling are redundant. This redundancy can be exploited to design opaque marker distribution schemes so that no information is lost on $g$ (only signal to noise is then lost).

In tomography, it is well known that the spectrum of the 2D Radon transform or the 3D x-ray transform has a particular geometry that can be exploited by interlaced sampling schemes $[16,13,6]$. In 2D tomography, we want to sample the Radon transform $g$ of an unknown function $f$ to be reconstructed

$$
g(\phi, s) \stackrel{\text { def }}{=} \int_{-\infty}^{\infty} f(s \theta+t \zeta) d t
$$

where $\theta=(\cos \phi, \sin \phi)$ and $\zeta=(-\sin \phi, \cos \phi)$. The Fourier transform of $g$ is defined by

$$
\hat{g}_{k}(\sigma)=\frac{1}{2 \pi} \int_{0}^{2 \pi} \hat{g}(\phi, \sigma) e^{-i k \phi} d \phi \text { with } \hat{g}(\phi, \sigma)=\frac{1}{\sqrt{2 \pi}} \int_{-\infty}^{\infty} g(\phi, s) e^{-i \sigma s} d s .
$$

It is shown $[16,13]$ that, if $f$ is essentially $b$ band limited, i.e., if $\int_{|\xi|>b}|\hat{f}(\xi)| d \xi<<$ $\int_{\mathbb{R}^{2}}|\hat{f}(\xi)| d \xi$, the set $\mathbf{K}_{2}$ is the essential support of $\hat{g}_{k}(\sigma)$, see figure 1 . Thus, the 
interlaced matrix $W_{I}$ satisfies the Shannon conditions with twice less points that the standard scheme $\left(\operatorname{det} W_{I}=2 \operatorname{det} W_{S}\right)$, with

$$
W_{S}=\left[\begin{array}{cc}
h_{1} & 0 \\
0 & h_{2}
\end{array}\right], W_{I}=\left[\begin{array}{cc}
2 h_{1} & -h_{1} \\
0 & h_{2}
\end{array}\right] \text {, }
$$

where $h_{1}=\frac{\pi}{p}, h_{2}=2 / q$ and $p \in \mathbb{N}$ is the projection number on $[0, \pi)$ and $q$ is the translation number on a diameter of the reconstruction disk (normalized to the unit disk in this paper). The sampling conditions are fullfilled if $p$ is slightly larger than $\pi q / 2=b$, see [13] and the figure 1 .

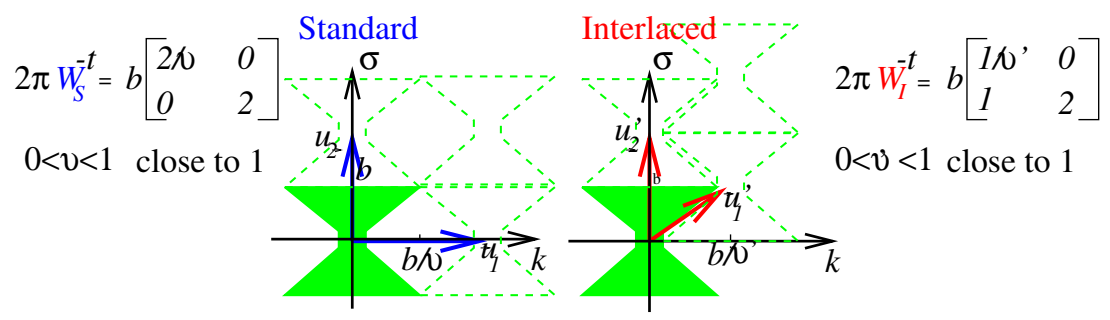

Fig. 1. Sampling condition of the 2D Radon transform in the Fourier space. The set $\mathbf{K}_{2}$ is shown in gray with translations on the reciprocal scheme generated by the matrix $\left(W_{I}^{-1}\right)^{t}$ (best interlaced scheme, right) and repectively $\left(W_{S}^{-1}\right)^{t}$ (best standard scheme, left). Note that the matrices given in (4) satisfy the sampling conditions if $h_{2}=\pi / b$ and $\pi / h_{1}=b / \vartheta^{\prime}$ (in practice $0<\vartheta^{\prime}<1$ close to 1 ).

In [6], these results have been generalized to 3D tomography. The measured function $f \in C_{0}^{\infty}(\Omega)$ is supposed to be essentially $b$ band limited where $\Omega$ is the unit cylinder of $\mathbb{R}^{3}$. The essential support of the Fourier transform $\hat{g}_{k}(\sigma, \tau)$ of the x-ray transform $g(\phi, s, t)$ is essentially $\mathbf{K}_{3}$-band limted (see figure 2) where

$g(\phi, s, t)=\int_{-\infty}^{\infty} f\left(s \theta+t e_{3}+u \zeta\right) d u$ and $\hat{g}_{k}(\sigma, \tau)=(2 \pi)^{-1} \int_{0}^{2 \pi} \hat{g}(\phi, \sigma, \tau) e^{-i k \phi} d \phi$, with $\zeta \in S^{1}$ (circle trajectory), i.e., $\phi \in[0,2 \pi], \zeta=(-\sin \phi, \cos \phi, 0), \theta=$ $(\cos \phi, \sin \phi, 0), e_{3}=(0,0,1), s \in[-1,1], t \in[-1,1]$, and where $\hat{g}(\phi, \sigma, \tau)$ is the Fourier transform of $g$ with respect to $s$ and $t$. This geometry is the classical parallel geometry. It corresponds to a $2 \mathrm{D}$ detector turning around the patient with a x-ray source at infinity. Once again, standard sampling grids are redundant and interlaced schemes are twice more efficient. Interlaced hexagonal schemes can be shown to be $4 / \sqrt{3}$ more efficient than the best standard grid.

Usual sampling schemes are standard grids. The main idea of this work is to place the calibration markers on a subset of the redundant part of the scheme. Indeed, if an interlaced scheme included in a standard scheme is not affected by the markers, all information in the 3D projection set can be essentially recovered thank to an interpolation formula based on (1). In the next section, we propose a fast interpolation formula for the $2 \mathrm{D}$ case. This can be generalized to $3 \mathrm{D}$. 

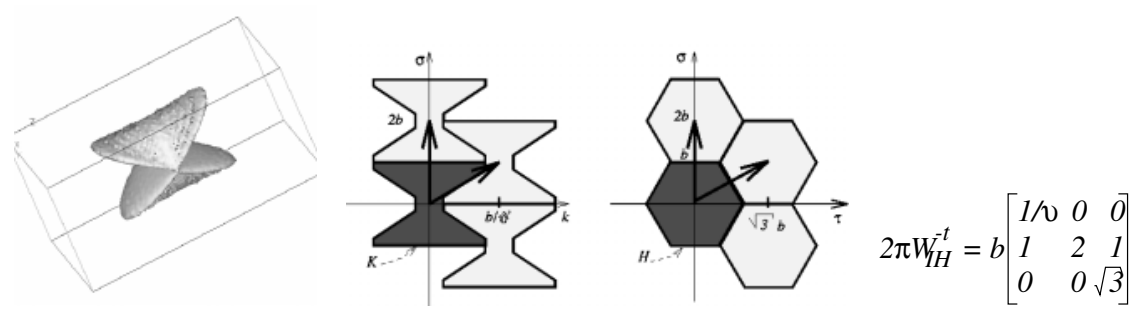

Fig. 2. left: $3 \mathrm{D}$ visualization of $\mathbf{K}_{3}$ : outside of this isosurface, $\left|\hat{g}_{k}(\sigma, \tau)\right|$ is negligible. Center and right: hexagonal interlaced non-overlapping sets $\mathbf{K}_{3}+2 \pi W_{I H}^{-t} l$ : $\mathbf{K}_{3}$ is contained at the intersection of two orthogonal cylinders of respective basis $K$ and the hexagon $H$.

\subsection{Exact Fourier Interpolation}

Interpolating the lacking data covered by markers allows applying (after interpolation) usual reconstruction methods based on standard schemes. Let us suppose that, in 2D, half of the standard points (in a sub-interlaced geometry see figure 3) are covered by calibration markers and thus are lacking. We want to estimate the complete data $g\left(W_{S} l\right), l \in \mathbb{Z}^{2}$ from the available sampling points $g\left(W_{I} l\right), l \in \mathbb{Z}^{2}$. Let us consider the Fourier transform of (1) (with $W=W_{I}$ )

$$
\hat{g}_{W_{I}}(\xi)=(2 \pi)^{-1}\left|\operatorname{det}\left(W_{I}\right)\right| \sum_{k \in \mathbb{Z}^{2}} g\left(W_{I} k\right) e^{-i \xi \cdot W_{I} k} \chi_{\mathbf{K}}(\xi) .
$$

From equation (5) $\hat{g}\left(\xi_{l}\right)$ with $\xi_{l}=2 \pi N^{-1} W_{S}^{-1} l, l \in \mathbb{Z}^{2}$ can be computed with DFT, where $N$ is a diagonal matrix $\operatorname{diag}\left(n_{1}, n_{2}\right)$. Indeed, a simple computation shows that (5) can be re-written

$$
\begin{aligned}
& \hat{g}_{W_{I}}\left(\xi_{l}\right)=(2 \pi)^{-1}\left|\operatorname{det}\left(W_{I}\right)\right| \chi_{\mathbf{K}}\left(\xi_{l}\right)\left\{\sum_{m \in \mathbb{Z}^{2}} g\left(W_{I}\left[\begin{array}{ll}
1 & 1 \\
0 & 2
\end{array}\right] m\right) e^{-2 i \pi\left(\frac{l_{1} m_{1}}{n_{1} / 2}+\frac{l_{2} m_{2}}{n_{2} / 2}\right)}\right. \\
& \left.+\sum_{m \in \mathbb{Z}^{2}} g\left(W_{I}\left[\begin{array}{ll}
1 & 1 \\
0 & 2
\end{array}\right] m+W_{I}\left[\begin{array}{l}
0 \\
1
\end{array}\right]\right) e^{-2 i \pi\left(\frac{l_{1} m_{1}}{n_{1} / 2}+\frac{l_{2} m_{2}}{n_{2} / 2}\right)} e^{-2 i \pi\left(\frac{l_{2}}{n_{2} / 2}-\frac{l_{1}}{n_{1} / 2}\right)}\right\} .
\end{aligned}
$$

In practice, the two previous sums are truncated, considering that the sinogram is periodic. Thus, with $m=\left(m_{1}, m_{2}\right)$ in $(6), m_{1}$ will be restricted to $0, \ldots, p$ and $m_{2}$ to $0, \ldots, q$ (the sinogram is periodic of period $2 \pi$ in its first variable and must be zero padded in the translation direction from $[-1,1]$ to $[-2,2])$. We will choose $n_{1} / 2=p$ and $n_{2}=q$. Thus, $\hat{g}_{W_{I}}\left(\xi_{l}\right)$ can be computed from two FFT of dimension $\left(n_{1} / 2, n_{2} / 2\right)$, respectively on the points $2 W_{S} \mathbb{Z}^{2}$ and $2 W_{S} \mathbb{Z}^{2}+W_{S}(-1,1)^{t}$. Indeed,

$$
W_{I}\left(m_{1}+m_{2}, 2 m_{2}\right)=2 W_{S}\left(m_{1}, m_{2}\right) \text { and } W_{I}(0,1)=W_{S}(-1,1) .
$$

The value of $g_{W_{I}}\left(W_{S} k\right)$ is obtained by inverse FFT on the $2 \mathrm{D}$ grid of $\hat{g}_{W_{I}}\left(\xi_{l}\right)$. In Figure 3 we show a numerical example of this approach based on (6). 

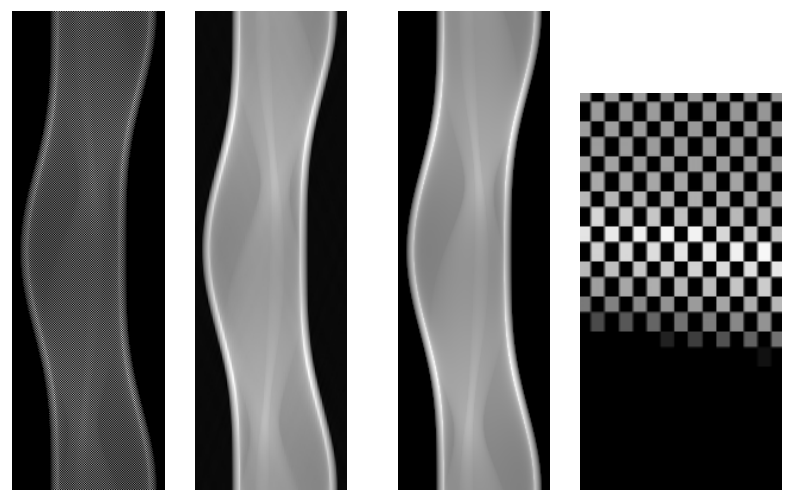

Fig. 3. From left to right: interlaced markers (see the zoom on the right) in a sinogram of $p=202$ projections on $[0, \pi[$ and $q=128$ samples ; Fourier interpolation from the available interlaced data ; corresponding complete standard sinogram ; zoom of a part of the sinogram on the left. We see that fast Fourier interpolation yields a good estimation of the complete sinogram, thus of the lacking data.

\subsection{D Reconstruction}

We can also directly reconstruct the function $f$ from the available data, i.e., not covered by markers. We have seen that they should yield as good reconstruction as from complete data, except from a (small) loss in signal to noise ratio.

We illustrate this approach in figure 4 using algebraic reconstruction techniques. Any kinds of reconstruction methods could be used from interpolated data, but algebraic methods can easily handle irregular geometry and thus avoid the interpolation step. The function $f$ is discretized in a sum of weighted voxel indicators. Then, the following regularized least squares problem is solved with a Conjugate Gradient algorithm:

$$
\min _{\mathbf{f}}\|\mathbf{A f}-\mathbf{g}\|^{2}+\lambda \mathbf{f}^{t} \Delta \mathbf{f}
$$

where $\mathbf{f}$ is the component vector of $f$ according to the voxels, $\mathbf{A}$ is an ARTlike matrix $\left(A_{i, j}\right.$ is the intersection length of the voxel $j$ with the projection line corresponding to the data $\left.g_{i}\right)$, $\mathbf{g}$ is the vector of data, $\Delta$ is the positive $3 \mathrm{D}$ Laplacian and $\lambda(>0)$ is a regularization parameter automatically chosen by generalized cross validation $[9,10]$.

The theoretical result on the spectrum of the data is established for parallel geometry, but it can be numerically verified for a conical transform for large radius of the circle path (let say greater than three time the radius of the cylindrical reconstruction region). 


\section{Discussion}

3D x-ray interventional radiology based on image intensifiers implies a dynamic distortion correction. In the case of multiple successive radiology acquired on a circle trajectory, we have shown that the spectral properties of the $3 \mathrm{D}$ data can be exploited to design marker schemes. Indeed, from the Shannon sampling theory, the whole information is then still contained in the data not overlapped by the interlaced markers. On the other hand, new generations of digital x-ray detectors [2], such as the PIXIUM 4600 from TRIXELL, do not suffer from distortions: 3D information on the patient (such as 3D reconstruction of bones surface) can be obtained without distortion correction. However, this very promising new technology is just emerging. Dynamic digital detector ( $>10$ images/s) are not available right now. Years will be needed before digital detectors will replace image intensifiers. That's why distortion correction technology remains of major interest.
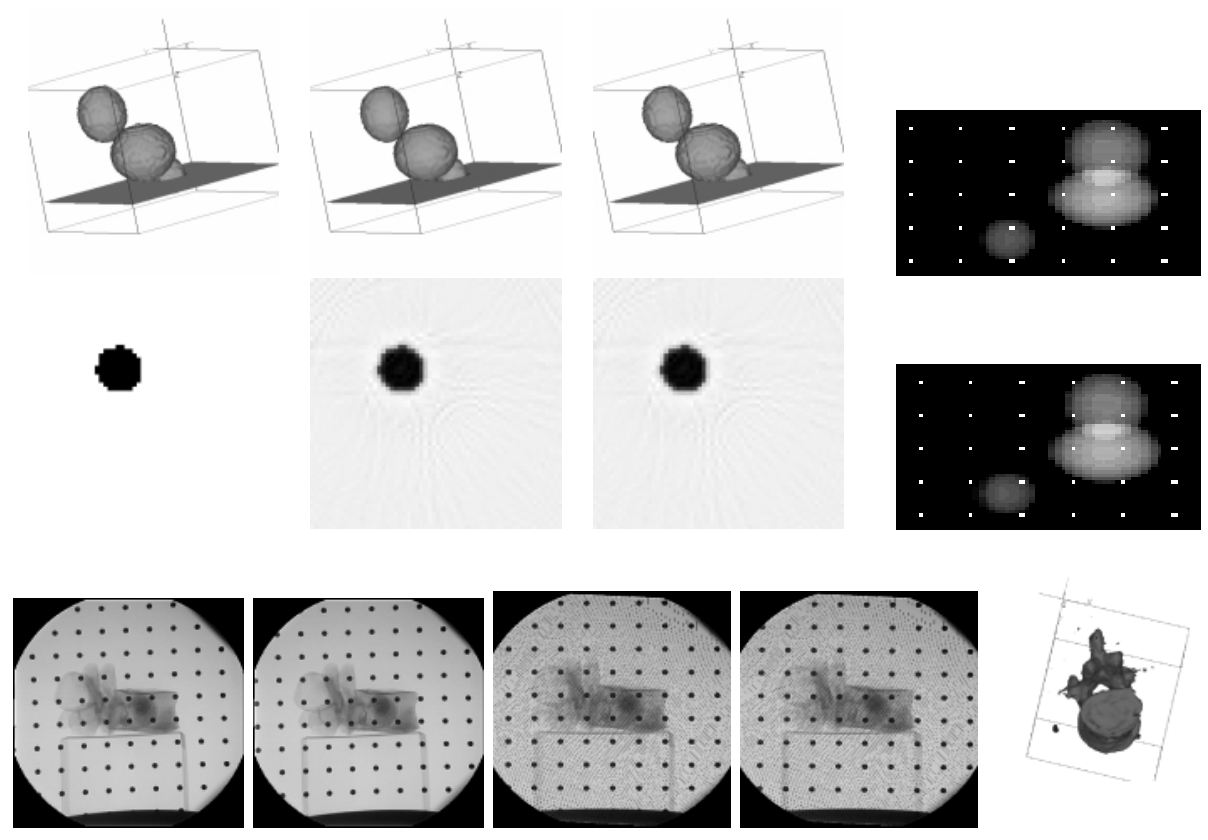

Fig. 4. Top, from left to right: object to be reconstructed; reconstruction from 90 projections with interlaced markers $(60 \times 50)$ on $[0, \pi]$; reconstruction from 90 projections without calibration markers; (right) an even projection with calibration markers. Second line: corresponding cross-section (3 first pictures); (right) an odd projection with translated calibration markers. Third line: even and odd projections of a vertebra; respectively after the distortion correction; 3D reconstruction of a vertebra from 63 projections on $[0 ; 2 \pi]$ with interlaced calibration markers. 


\section{Acknowledgments}

This work is supported by a grant of the Région Rhône-Alpes within the project "Santé et HP : de la Vision au Pilotage".

\section{References}

1. G. Champleboux, S. Lavallee, P. Sautot, and P. Cinquin. Accurate calibration of cameras and range imaging sensors, the NPBS method. In IEEE Int. Conf. on Robotics and Automation, pages 1552-1558, Nice France, May 1992. 974

2. C. Chaussat, J. Chabbal, T. Ducourant, V. Spinnler, and G. Vieux. New superior detectivity $\mathrm{CsI} / \mathrm{a}-\mathrm{Si} 43 \mathrm{~cm}$ x $43 \mathrm{~cm}$ x-ray flat panel detector for general radiography provides immediate direct digital output and easy interfacing to digital radiographic systems. In H.U. Lemke, editor, CAR, 1998. 979

3. O. Chavanon, C. Barbe, J. Troccaz, L. Carrat, C. Ribuot, and D. Blin. Computer ASsisted PERicardial punctures : work in progress. Computer Aided Surgery, 2(6):356-364, 1997.974

4. J.L. Coatrieux and C. Roux. Biomedical imaging: integration of image engineering biology and medicine. In Contenporary perspectives in three-dimensional biomedical imaging, chapter 1, pages 3-27. IOS Press, 1997. 974

5. M. Defrise and R. Clack. Cone-beam reconstruction using shift variant filtering and cone beam backprojection. IEEE Trans. MI, 13:186-195, 1994. 974

6. L. Desbat. Echantillonnage parallèle efficace en tomographie 3D. C.R. Acad. Sci. Paris, Série I, t. 324, pages 1193-1199, 1997. 975, 976

7. I.A. Feldkamp, L.C. Davis, and J.W. Kress. Practical cone-beam algorithm. J. Opt. Soc. Am. A, 1(6):612-619, 1984. 974

8. M. Fleute and S. Lavallee. Nonrigid 3-D/2-D Registration of Images Using Statistical Models. In MICCAI'99, 1999. accepted. 974

9. D.A. Girard. A fast Monte Carlo cross-validation procedure for large least squares problems with noisy data. Numer. Math., 56:1-23, 1989. 978

10. D.A. Girard. Asymptotic optimality of the fast randomized versions of GCV and $\mathrm{C}_{L}$ in ridge regression and regularisation. Ann. of Stat., 19(4):1950-1963, 1991. 978

11. P. Grangeat. Reconstruction d'images tridimensionnelles. INPG, 1993. Thèse d'habilitation à diriger des recherches. 974

12. A. Hamadeh, P. Sautot, S. Lavallee, and P. Cinquin. Towards automatic registration between $\mathrm{CT}$ and X-ray images : cooperation between $3 \mathrm{D} / 2 \mathrm{D}$ registration and 2D edge detection. In Second Symposium on Medical Robotics and Computer Assisted Surgery Proc. (MRCAS'95), pages 39-46, Baltimore, MA, nov. 1995. Wiley. 974

13. F. Natterer. The Mathematics of Computerized Tomography. Wiley, 1986. 975, 976

14. F. Noo, M. Defrise, and R Clackdoyle. Single-slice rebinning method for helical con-beam CT. Phys. Med. Biol., 44:561-570, 1999. 974

15. C. Pellot, A. Herment, M. Sigelle, P. Horin, H. Maître, and P. Peronneau. 3D reconstruction of vascular structures from 2 X-ray angiograms using an adapted simulated annealing algorithm. IEEE trans. Med. Im., 13(1):48-60, 1994. 974

16. P.A. Rattey and A.G. Lindgren. Sampling the 2-D Radon transform. IEEE Trans. ASSP, 29:994-1002, 1981. 975 